\title{
Comparison of Real-time Polymerase Chain Reaction Results by Different Illumination Methods
}

\author{
Ji-Soo Hwang, ${ }^{1,2}$ Jong-Dae Kim, ${ }^{2,3}$ Yu-Seop Kim, ${ }^{2,3}$ \\ Hye-Jeong Song, ${ }^{2,3}$ and Chan-Young Park ${ }^{2,3^{*}}$ \\ ${ }^{1}$ Department of Computer Engineering, Hallym University, \\ 1, Hallimdaehak-gil, Chuncheon-si, Gangwon-do 24252, Republic of Korea \\ ${ }^{2}$ Department of Convergence Software, Hallym University, \\ 1, Hallimdaehak-gil, Chuncheon-si, Gangwon-do 24252, Republic of Korea \\ ${ }^{3}$ Bio-IT Research Center, Hallym University, \\ 1, Hallimdaehak-gil, Chuncheon-si, Gangwon-do 24252, Republic of Korea
}

(Received July 30, 2017; accepted November 9, 2017)

Keywords: polymerase chain reaction, fluorescence detection test, PCR chip, lab on a chip, illumination method, real-time PCR, quantitative analysis

In this paper, we introduce the diagonal illumination method and the vertical illumination method of the real-time polymerase chain reaction (PCR) system and compare the fluorescence intensity change patterns of the two methods using images captured with a digital single lens reflex (DSLR) camera during the PCR process. To overcome the problems of diagonal illumination, the PCR was performed by vertical illumination. The results of the PCR process with the proposed vertical illumination method were similar to those of the diagonal illumination method.

\section{Introduction}

Polymerase chain reaction (PCR) is a molecular biology technique that amplifies small amounts of gene samples to large quantities to detect or sense DNAs. It is used to amplify human DNA and diagnose various genetic diseases. It can be applied to bacterial, viral and fungal DNA to enable diagnosis of infectious diseases. In the conventional PCR method, the result of DNA amplification is verified by electrophoresis. However, in the real-time PCR method, DNA amplification products are monitored in real time and quantitatively analyzed. At this time, the intensity of the signal detected by fluorescence is used to analyze the result.

The real-time PCR chip proposed in the previous study consisted of a polycarbonate plastic cover on a coated matte black printed circuit board (PCB) substrate, packing tape, black doublesided tape, and a white silk legend. DNA amplification tests using these chips were successful. ${ }^{(1-4)}$ For the fluorescence detection presented in the previous study, the blue LED provided diagonal

*Corresponding author: e-mail: cypark@hallym.ac.kr http://dx.doi.org/10.18494/SAM.2018.1760 
illumination and the fluorescence intensity values of the images captured by the digital single lens reflex (DSLR) camera during the polymerase chain reaction were calculated and plotted as the sum of means, medians, and bhattachryya distances. ${ }^{(3)}$ Although this illumination method is simple, there are problems: the intensity of light changes depending on slight differences between the distance and the angle of illumination, and it is spatially limited by the apparatus used, such as a camera or an optical apparatus for fluorescence detection. Therefore, in this paper we propose a vertical illumination method. Since this method attaches a small surfacemount device (SMD)-type LED vertically to the PCR chip cover, the angle and distance problems can be alleviated and the size of the detection device can be reduced. In order to observe the changes in fluorescence intensity of the images captured by a DSLR camera during the PCR process using the two illumination methods, the sum-of-means values were calculated using Matlab and the patterns of brightness change were compared.

\section{Materials and Methods}

\subsection{Chip driving system}

Figure 1 shows a block diagram of proposed real-time PCR control system. The control system consists of a local system and the PCR chip. The thermistor of the PCR chip reports a digital temperature value calculated from the voltage divider and analog to digital converter values. A USB port connects the local system to a PC. The temperature is recorded in the local system and is sent to the host PC every $50 \mathrm{~ms}$. At the host, the temperature data and the pulsewidth modulation (PWM) value are derived from a proportional-integral-derivative (PID) controller mechanism. ${ }^{(5,6)}$

The PCR protocol set by the user on the PC is transferred to the local system and executed. The local system activates the heater and cooling fan to maintain the temperature set in the PCR protocol for a specified time. During the amplification of the DNAs, the LED is illuminated at a specific time in the extension step of the PCR protocol, and the fluorescence of the sample is measured using a camera. ${ }^{(7-10)}$

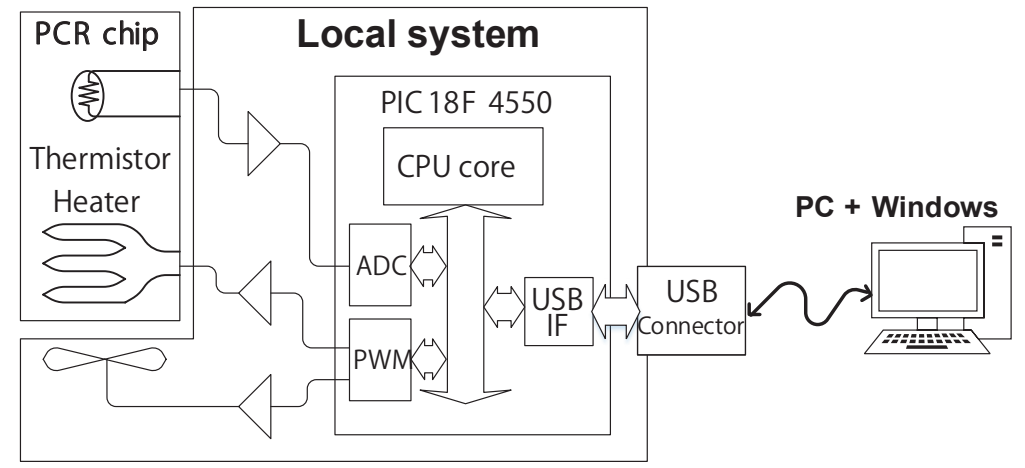

Fig. 1. Block diagram of the proposed real-time PCR system. 


\subsection{PCR chip structure}

The micro-PCR chip has a six-layer structure that contains a matte black PCB, packing tape, black double-sided tape, a plastic cover, the housing, and transparent double-sided tape to connect the housing with the black PCB. Heating circuits are attached at the very bottom of the PCB base and a thermistor for sensing temperature.

To prevent the fluorescent substance from sticking to the PCB, packing tape made of polypropylene material was attached to the top of the PCB. Since the actual tube used in the biology laboratory was also made of polypropylene, the same material was also used for the chip to create an experimental environment similar to the centrifuge tube. To set the height of the microfluidic channel to $400 \mu \mathrm{m}, 200-\mu \mathrm{m}$-thick black double-sided tape was attached in two layers.

Table 1 shows the tapes used in the experiment. Packing tape and double-sided tape were used in the chip, with the double-sided tape serving an important role in the channel of the micro-PCR chip. As previously reported, it can withstand high temperatures of $95{ }^{\circ} \mathrm{C}$ and high pressures when expanding. The adhesive was acrylic, and the carrier of the double sided tape was composed of PET. The chamber in the middle in which the PCR was performed was fabricated by drilling a U-shaped hole. ${ }^{(3)}$

The cover at the top part of the chip was made with $200-\mu \mathrm{m}$-thick polycarbonate material. Lastly, the PCB and the upper structure were fixed in the housing, and the tiny channel of this housing was used as a passageway for the reagent injection and the extraction of PCR products.

\subsection{Experimental method}

Figure 2 shows the fluorescence detection measurement apparatus used in the experiment. Result images were captured with a DSLR camera. To detect fluorescein amidite (FAM) fluorescence, a blue LED (9600 mcd) was illuminated diagonally for diagonal illumination and a small surface-mount device (SMD) type blue LED $(100 \mathrm{mcd})$ was illuminated vertically from the top of the cover to the reaction chamber in order to overcome the problems of the diagonal illumination method. The chip used in the experiment was made by integrating the chip and the illumination source by attaching the small blue LED on the cover of the chip and those of the PCR test results were compared with the previously proposed diagonal illumination method shown in Fig. 2(b). The PCB was fabricated into a structure that allows for easy observation of an increase or decrease of fluorescence intensity owing to a white silk legend printed on the

Table 1

Packing and double-sided tapes used in the experiment.

\begin{tabular}{|c|c|c|c|}
\hline & Manufacturer & Tape number & Adhesive type \\
\hline Packing tape & $3 \mathrm{M}$ & \#309 Mini Clear & Acrylic adhesive \\
\hline Double-sided tape & Nitto Denko (Japan) & $\begin{array}{c}\text { No. 5620BWN } \\
\text { (thickness } 0.2 \mathrm{~mm} \text { ) }\end{array}$ & High adhesion acrylic \\
\hline Double-sided tape & Nitto Denko (Japan) & $\begin{array}{c}\text { No. } 5620 \\
\text { (thickness } 0.2 \mathrm{~mm} \text { ) }\end{array}$ & High adhesion acrylic \\
\hline
\end{tabular}




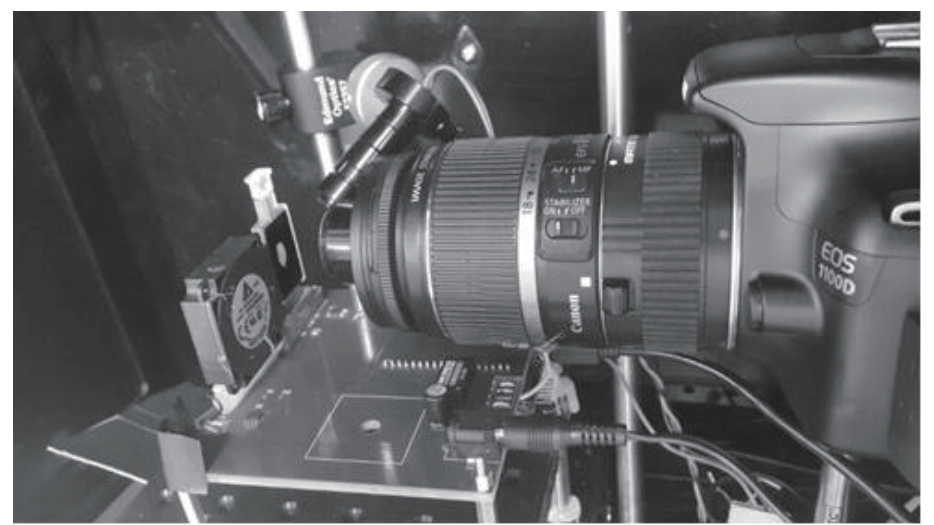

(a)

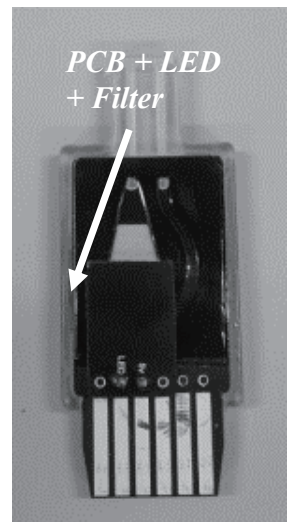

(b)

Fig. 2. (a) Fluorescence detection apparatus and (b) one chip PCR emulation module.

chamber. The chip cover was thinner than that previously used to increase the transmittance of the LED light and reduce the reflectance.

The PCR process was performed according to the following protocol. The temperature was controlled by the following PCR protocol consisting of three steps: denaturation, annealing, and extension. DNA amplification was performed for a total of 40 cycles. The PCR protocol was carried out for $10 \mathrm{~s}$ at $95{ }^{\circ} \mathrm{C}, 30 \mathrm{~s}$ at $60{ }^{\circ} \mathrm{C}$, and $30 \mathrm{~s}$ at $72{ }^{\circ} \mathrm{C}$, after pre-denaturation treatment at $95^{\circ} \mathrm{C}$ for $3 \mathrm{~min}$ as shown in Table 2 .

The reagents used for PCR were $1 \mathrm{ng}$ DNA (Chlamydia trachomatis) in $15.84 \mu \mathrm{L}$ solution, $18 \mu \mathrm{L}$ Master Mix, $0.72 \mu \mathrm{L}$ of $10 \mathrm{pM}$ primer F, $0.72 \mu \mathrm{L}$ of $10 \mathrm{pM}$ primer $\mathrm{R}$ and $0.72 \mu \mathrm{L}$ of $10 \mathrm{pM}$ probe respectively (the total volume was $36 \mu \mathrm{L}$ ).

The experiment was conducted using the fluorescence detection setup shown in Fig. 2(a), and images were automatically captured with a DSLR camera whenever a cycle reached $72{ }^{\circ} \mathrm{C}$ (the extension step of nucleic acid amplification).

\section{Experiments and Results}

Figure 3(a) shows the analysis of the real-time PCR results by the diagonal illumination method. Fluorescent brightness values of 40 images captured using a DSLR camera were analyzed using a fluorescence intensity analysis program made with Matlab.

The color images were transformed into grayscale and the chamber area was masked to calculate the sum of means, medians, and bhattacharyya distances, which are shown in Fig. 3(a). The sum of means is the most commonly used method to compare brightness.

Figure 3(b) shows the result of PCR performed by the vertical illumination method. Unlike the diagonal illumination method, the masking area was manually determined, the images were converted to grayscale, and the sum of means was calculated and plotted as shown in the figure.

As shown in Figs. 3(a) and 3(b), fluorescence brightness decreased or remained almost unchanged until cycles 11-12, however, the results indicate a sharp exponential increase from cycles 12-13. In each experiment, the intensities of the fluorescence intensity were different, but the pattern of the change in fluorescence brightness was similar, as shown in the two plots. 
Table 2

The PCR protocol used in the experiment.

\begin{tabular}{lcc}
\hline PCR process & Temperature $\left({ }^{\circ} \mathrm{C}\right)$ & Time duration \\
\hline 1 cycle & 95 & $3 \mathrm{~min}$ \\
\hline \multirow{3}{*}{40 cycles } & 95 & $10 \mathrm{~s}$ \\
& 60 & $30 \mathrm{~s}$ \\
\hline \multirow{2}{*}{1 cycle } & 72 & $30 \mathrm{~s}$ \\
& 95 & $10 \mathrm{~s}$ \\
& 50 & $30 \mathrm{~s}$ \\
\hline
\end{tabular}

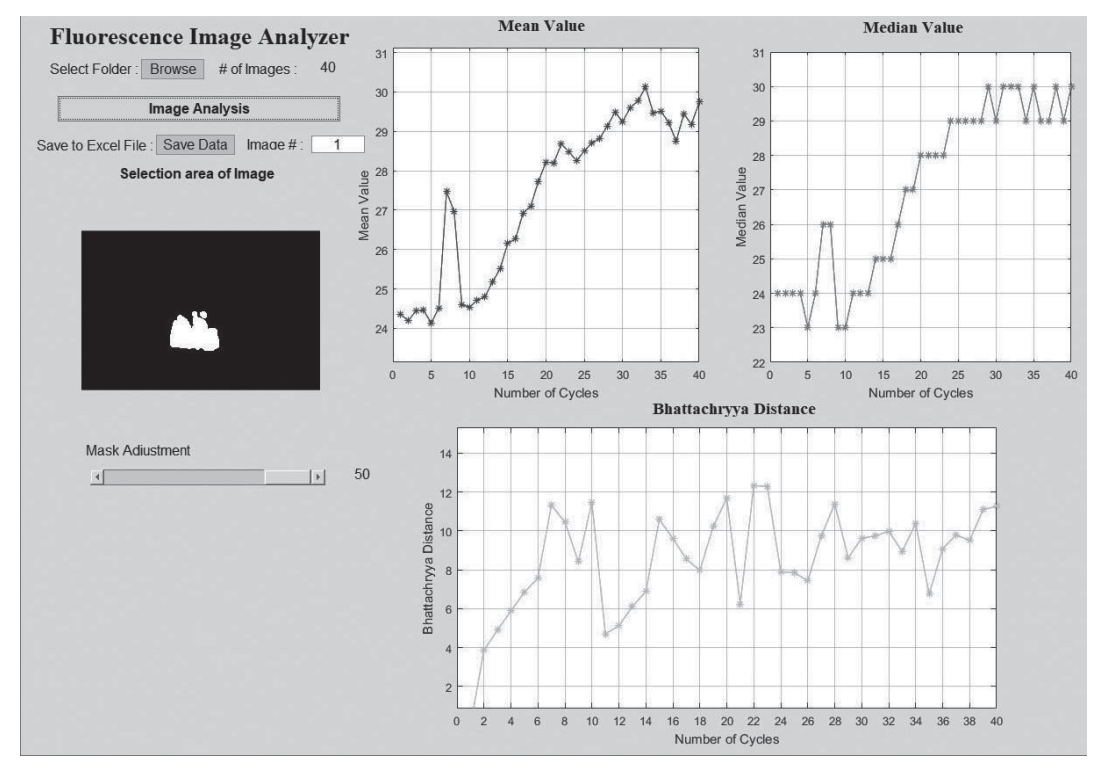

(a)

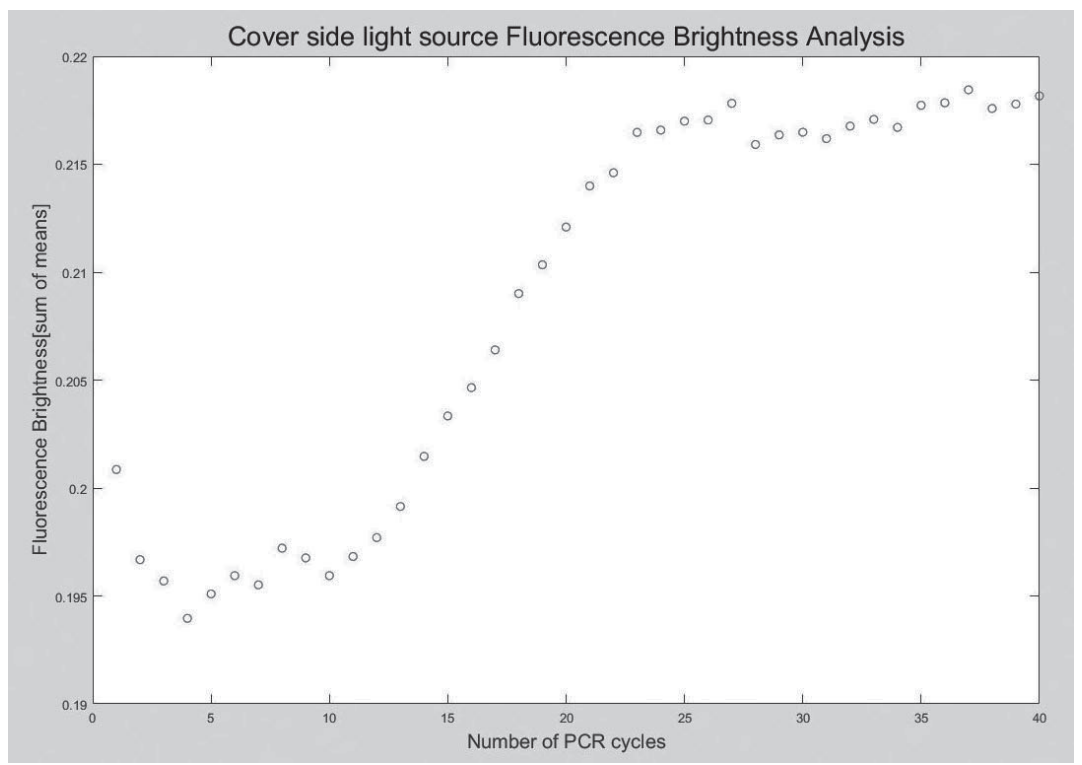

(b)

Fig. 3. Fluorescence detection test results by (a) diagonal and (b) vertical illumination methods. 


\section{Conclusions}

In this paper, we compared two illumination methods for fluorescence detection in real-time PCR. One is the previously reported diagonal illumination method and the other is a downward vertical illumination method achieved by fixing the LED module on the PCR chip cover. The PCR chip was made by integrating the chip and the illumination by attaching a small blue LED on the cover of the chip.

The previously reported diagonal illumination method is very simple, but there are problems in that the intensity changes sensitively according to the distance or the illumination angle, and it is subject to space limitation due to the camera or optical device that is required for fluorescence detection.

In this experiment, the results from the two methods show similar pattern types of the sum of means of fluorescence brightness. This means that fluorescence detection is possible even with illumination vertically downward from the top of the chip cover.

The vertical illumination method is not spatially limited and ensures a constant illumination intensity. In addition, since the size of the LED is reduced, it is expected that the total system can be reduced in size.

\section{Acknowledgments}

This research was supported by the National Research Foundation of Korea (NRF) funded by the Ministry of Science, ICT and Future Planning (NRF-2017R1A2B1010125).

\section{References}

1 D. Patko, Z. Mártonfalvi, B. Kovacs, F. Vonderviszt, M. Kellermayer, and R. Horvath: Sens Actuators, B 196 (2014) 352 .

2 M. Focke, D. Kosse, C. Müller, H. Reinecke, R. Zengerle, and F. von Stetten: Lab Chip 10 (2010) 1365.

3 J. S. Hwang, Y. S. Kim, H. J. Song, J. D. Kim, and C. Y. Park: Technol. Health Care 24 (2016) S139.

4 J. S. Hwang, S. Y. Kim, Y. S. Kim, H. J. Song, C. Y. Park, and J. D. Kim: Int. J. Control Autom. 8 (2015) 117.

5 C. Plaisant, A. Rose, B. Shneiderman, and A. J. Vanniamparampil: IEEE Software 14 (1997) 66.

6 C. E. Wills: Electro/94 Int. Conf. Proc. Combined Volumes (1994) 415.

7 J. Kim, J. Kim, and G. Lee: SPIE MOEMS-MEMS (2011) 79290Y.

8 C. Y. Park, J. D. Kim, J. H. Ku, Y. S. Kim, H. J. Song, and J. W. Kim: Sens. Lett. 10 (2012) 1197.

9 T. M. Hsieh, C. H. Luo, F. C. Huang, J. H. Wang, L. J. Chien, and G. B. Lee: Sens. Actuators, B 130 (2008) 848.

10 K. Shen, X. Chen, M. Guo, and J. Cheng: Sens. Actuators, B 105 (2005) 251. 